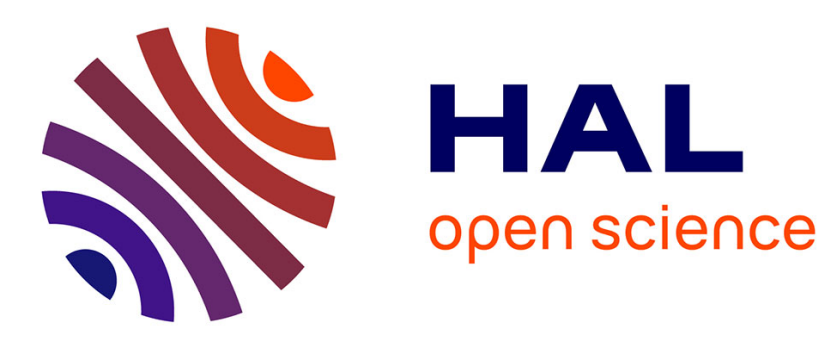

\title{
Distributions énergétiques des ions secondaires
}

\author{
G. Blaise, G. Slodzian
}

\section{To cite this version:}

G. Blaise, G. Slodzian. Distributions énergétiques des ions secondaires. Revue de Physique Appliquée, 1973, 8 (2), pp.105-116. 10.1051/rphysap:0197300802010500 . jpa-00243663

\section{HAL Id: jpa-00243663 https://hal.science/jpa-00243663}

Submitted on 1 Jan 1973

HAL is a multi-disciplinary open access archive for the deposit and dissemination of scientific research documents, whether they are published or not. The documents may come from teaching and research institutions in France or abroad, or from public or private research centers.
L'archive ouverte pluridisciplinaire HAL, est destinée au dépôt et à la diffusion de documents scientifiques de niveau recherche, publiés ou non, émanant des établissements d'enseignement et de recherche français ou étrangers, des laboratoires publics ou privés. 
Classification

Physics Abstracts

16.95

\title{
DISTRIBUTIONS ÉNERGÉTIQUES DES IONS SECONDAIRES
}

\author{
G. BLAISE et G. SLODZIAN
}

Laboratoire de Physique des Solides associé au CNRS

Bât. 510, Université Paris-Sud-Centre d'Orsay, France

(Reçu le 24 octobre 1972)

\begin{abstract}
Résumé. - Les distributions énergétiques des ions secondaires ont été analysées par une méthode de contre-champ au moyen du miroir électrostatique qui équipe le micro-analyseur ionique. Les résultats présentés concernent essentiellement les ions $\mathbf{M}^{+}$et $\mathbf{M}_{n}^{+}$provenant de métaux et d'alliages bombardés par des ions primaires $\mathbf{A}^{+}$. L'accent est mis sur les aspects très différents des distributions des ions de faible énergie, inférieure à $20 \mathrm{eV}$ environ.
\end{abstract}

Abstract. - The energy distributions of secondary ions are analysed with the help of the electrostatic mirror that equips the ion microanalyser. The results concern primarely $\mathbf{M}^{+}$and $\mathbf{M}_{\mathbf{n}}^{+}$ ions emitted by pure metals and alloys bombarded with $\mathrm{A}^{+}$primary ions. Differences in the low energy range of the distributions, below $20 \mathrm{eV}$, are demonstrated.

1. Introduction. - Les ions secondaires qui sont émis au cours de la pulvérisation des métaux présentent des dispersions énergétiques importantes.

L'étude des distributions énergétiques a souvent été menée à l'aide de dispositifs de filtrage adaptés sur des spectromètres de masse [1], [2]. Cependant, la résolution insuffisante de ces dispositifs ne permettait pas de relever la forme des distributions en énergie des ions en dessous d'une dizaine d'électrons-volts environ. Or, une connaissance précise de la distribution énergétique des ions les plus lents peut apporter des informations utiles pour la compréhension des processus d'ionisation. En effet, si l'on admet que la formation d'un état auto-ionisant sur une particule qui sort du métal est à l'origine de l'émission ionique [3], la survie de cet état (jusqu'au moment où cesse tout échange avec le métal) peut dépendre de la vitesse d'éjection. C'est précisément dans le domaine d'émission des ions de basse énergie que cette dépendance sera la plus sensible.

Avec le miroir électrostatique qui équipe le microanalyseur ionique [4], on dispose d'un moyen bien adapté à l'étude des distributions énergétiques puisqu'il permet une analyse fine par contre-champ dans des conditions optiques bien définies.

2. Méthode expérimentale. - 2.1 DESCRIPTION DU MICRO-ANALYSEUR IONIQUE (Fig. 1). - La pulvérisation de l'échantillon est assurée par des ions $\mathrm{A}^{+}$qui arrivent sous une incidence de $60^{\circ}$ environ et dont l'énergie est de $6,2 \mathrm{keV}$ au moment de l'impact. Dans nos conditions expérimentales, la densité de ce faisceau de bombardement est de l'ordre de $10 \mu \mathrm{A} / \mathrm{mm}^{2}$.

Les ions secondaires qui proviennent de l'objet sont tout d'abord accélérés sous une différence de potentiel $V=3800 \mathrm{~V}$, puis focalisés. Un diaphragme C, placé au cross-over de sortie du système de focalisation opère un filtrage suivant l'énergie latérale des ions. On montre (5) qu'un ion secondaire d'énergie cinétique $E_{\mathrm{c}}$ franchit le diaphragme $\mathrm{C}$ de diamètre $d$ si la direction de sa vitesse, au moment de son éjection, fait un angle inférieur à un angle limite $\alpha_{\mathrm{m}}$ qui dépend de $E_{\mathrm{c}}$. L'angle $\alpha_{\mathrm{m}}$ est donné par

$$
\begin{array}{rlrl}
\alpha_{\mathrm{m}} & =\frac{\pi}{2} & \text { pour } & E_{\mathrm{c}} \leqslant E_{0} \\
\sin \alpha_{\mathrm{m}}=\frac{E_{0}}{E_{\mathrm{c}}} & \text { pour } & E_{\mathrm{c}}>E_{0}
\end{array}
$$

avec

$$
E_{0}=\frac{q}{e} V\left(\frac{d}{8 \Delta}\right)^{2}
$$

où $\Delta$ est la distance entre l'objet et l'électrode d'accélération, $q$ la charge de l'ion, $e$ la charge élémentaire. Les énergies $E_{0}$ et $E_{\mathrm{c}}$ qui figurent dans les relations (1) ainsi que dans les expressions suivantes (2) et (3) sont exprimées en électrons-volts.

Dans les conditions suivantes $V=3800 \mathrm{~V}$, $\Delta=3,8 \mathrm{~mm}, d=0,4 \mathrm{~mm}$ et $q / e=1$, on a $E_{0} \sim 0,7 \mathrm{eV}$.

Une partie des ions est donc éliminée au niveau du diaphragme C. En toute rigueur, la détermination 
de l'efficacité de la collection exige la connaissance préalable des distributions énergétique et angulaire dans les conditions particulières de l'expérience. Mais si l'on suppose que la distribution angulaire se fait suivant une loi de Lambert quelle que soit l'énergie des particules secondaires, l'émission est entièrement caractérisée par la fonction de distribution $n\left(E_{\mathrm{c}}\right)$ telle que, $\mathrm{d} N_{\mathrm{i}}=n\left(E_{\mathrm{c}}\right) \mathrm{d} E_{\mathrm{c}}$ donne le nombre $\mathrm{d} N_{\mathrm{i}}$ d'ions émis dans l'intervalle d'énergie $E_{\mathrm{c}}, E_{\mathrm{c}}+\mathrm{d} E_{\mathrm{c}} ; n\left(E_{\mathrm{c}}\right)$ étant relié au nombre d'ions $n_{\mathrm{D}}\left(E_{\mathrm{c}}\right)$ qui franchissent $\mathrm{C}$ par les relations (2):

$$
\begin{array}{lll}
n_{\mathrm{D}}\left(E_{\mathrm{c}}\right)=n\left(E_{\mathrm{c}}\right) & \text { pour } & E_{\mathrm{c}} \leqslant E_{0} \\
n_{\mathrm{D}}\left(E_{\mathrm{c}}\right)=n\left(E_{\mathrm{c}}\right) \frac{E_{0}}{E_{\mathrm{c}}} & \text { pour } & E_{\mathrm{c}}>E_{0} .
\end{array}
$$

Il est donc possible, dans ce cas, de remonter assez simplement au nombre $n\left(E_{\mathrm{c}}\right)$ d'ions émis. La distribution angulaire des ions ne suit certainement pas une loi de Lambert (2). Cependant, si l'on cherche seulement à corriger la discrimination introduite par le diaphragme, on peut montrer (6) que l'approximation faite en supposant que la fraction des ions transmis est égale à $E_{0} / E_{\mathrm{c}}$ n'introduit qu'une erreur de quelques pour-cent sur le nombre $n\left(E_{\mathrm{c}}\right)$ d'ions qu'on obtiendrait à partir de la distribution angulaire réelle.

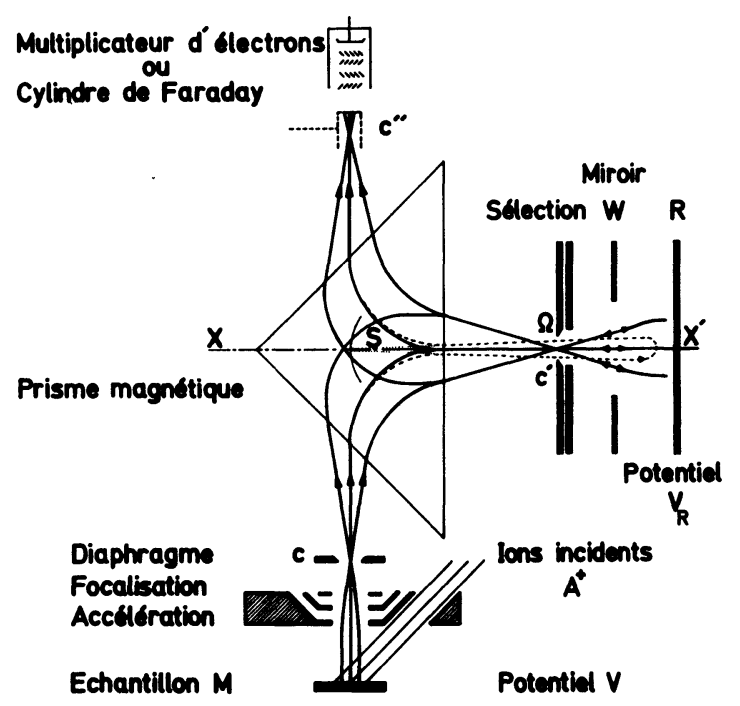

Fig. 1. - Micro-analyseur ionique à double déflexion magnétique équipé d'un miroir électrostatique.

Le diaphragme $\mathrm{C}$ délimite la pupille d'entrée du prisme magnétique qui sert à l'analyse du faisceau. Ce prisme offre la particularité de focaliser les ions dans les deux directions de l'espace de sorte que le faisceau issu de $\mathrm{C}$ converge, après rotation de $90^{\circ}$, en $C^{\prime}$. Une fente de sélection placée en $C^{\prime}$ permet de filtrer les ions suivant leur quantité de mouvement : pour des ions de même masse, cette fente délimite donc une bande d'énergie. Le miroir électrostatique est disposé de telle sorte que son centre $\Omega$ coïncide avec $\mathrm{C}^{\prime}$ et que son sommet $\mathrm{S}$ se trouve à peu près sur la focale achromatique de la première traversée du prisme [5]. Les ions qui ont franchi la fente de sélection et qui pénètrent dans le miroir avec une vitesse trop grande pour être réfléchis viennent heurter l'électrode répulsive $\mathrm{R}$; ils perdent alors leur charge électrique et disparaissent du faisceau. Les autres sont soumis, au cours de leur réflexion, à l'action convergente du miroir et forment à la sortie un faisceau divergent de sommet $\mathrm{C}^{\prime}$; ils se dirigent vers le prisme magnétique où ils subissent une nouvelle déviation, symétrique de la première par rapport à l'axe horizontal $\mathrm{x}^{\prime} \mathrm{x}$ de la figure 1 . Par suite de la symétrie du montage, les trajectoires convergent maintenant en $\mathrm{C}^{\prime \prime}$, symétrique de $\mathrm{C}$ par rapport à $\mathrm{x}^{\prime} \mathrm{x}$. La position du sommet $\mathrm{S}$ est telle que les ions réfléchis sont tous focalisés en $\mathrm{C}^{\prime \prime}$, bien qu'ils aient des énergies différentes. On mesure le courant $I_{\mathrm{r}}$ des ions réfléchis, au niveau de $\mathrm{C}^{\prime \prime}$, en interposant un cylindre de Faraday ou un multiplicateur d'électrons.

2.2 Filtrage DU Miroir (Fig. 2). - Considérons un faisceau réduit à sa trajectoire centrale parcourue par des ions d'énergie $q \varphi$. Lorsque cette trajectoire est confondue avec l'axe $x^{\prime} x$, les ions entrent dans le miroir, s'arrêtent sur l'équipotentielle $\varphi$ et repartent en sens inverse. Pour un réglage légèrement différent de

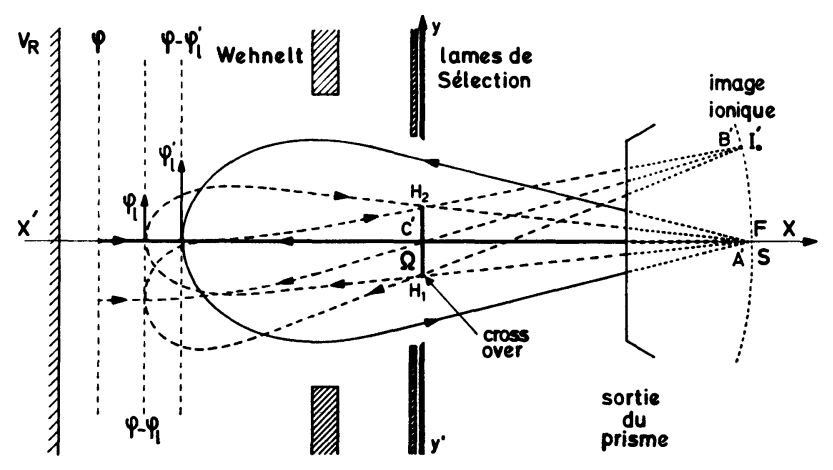

FIG. 2. - Filtrage du miroir électrostatique.

- En traits pleins : trajectoires de références suivies par des ions d'énergie $q \varphi$, pour différentes valeurs de l'induction magnétique. - En traits pointillés : trajectoires des ions d'énergie $q \varphi$ lorsque la trajectoire de référence est centrée sur l'axe du miroir, que le sommet est réglé sur la focale achromatique et que la lentille à émission est réglée de manière à amener l'image de l'objet sur le sommet du miroir.

l'induction magnétique, ces ions sortent du prisme en suivant une trajectoire rectiligne, inclinée sur $x^{\prime} x$ et qui s'appuie sur la focale achromatique $F$. Le miroir est réglé de telle sorte que son sommet $\mathrm{S}$ soit placé en $\mathrm{F}$. Il en résulte que la trajectoire réfléchie est symétrique de la trajectoire incidente par rapport à $x^{\prime} x$ et qu'elle repasse par $F$. Mais la « réflexion » s'effectue maintenant sur l'équipotentielle $\varphi-\varphi_{1}^{\prime}$, car au moment où la composante de la vitesse suivant $\mathrm{x}^{\prime} \mathrm{x}$ s'annule, il subsiste une énergie $q \varphi_{1}^{\prime}$ du fait que l'ion possède encore une composante de vitesse perpendiculaire à $\mathrm{x}^{\prime} \mathrm{x}$. Du point de vue optique, tout se passe comme 
si l'on avait une lentille à émission dont la surface émissive, confondue avec l'équipotentielle $\varphi-\varphi_{1}^{\prime}$, laisserait partir, tangentiellement, un ion d'énergie $q \varphi_{1}^{\prime}[5]$.

En définitive, lorsque le potentiel $V_{\mathrm{R}}$ de l'électrode répulsive $\mathrm{R}$ est égal à $\varphi$ et que l'induction est réglée de telle sorte que la trajectoire centrale suive l'axe $\mathrm{x}^{\prime} \mathrm{x}$, les ions d'énergie $q \varphi$ qui entrent dans le miroir touchent l'électrode $\mathrm{R}$ et sont neutralisés. Par contre, pour un réglage différent de l'induction, ces mêmes ions sont réfléchis sur l'équipotentielle $\varphi-\varphi_{1}^{\prime}$. Il faut donc examiner d'un peu plus près l'optique du prisme associé au miroir pour savoir l'effet qui en résultera sur le filtrage en énergie.

2.2.1 Faisceau d'ions monocinétiques. - Imaginons, pour simplifier, que la lentille initiale du micro-analyseur donne de la surface de l'objet une image $I_{0}^{\prime}$ située en $S$ (Fig. 2) ; le miroir laissera $I_{0}^{\prime}$ inchangé. Chaque point de cette image est éclairé par la pupille $C^{\prime}$, située en $\Omega$, dont la dimension dans le plan de la figure 2 est donnée par $\mathrm{H}_{1} \mathrm{H}_{2}$. Supposons encore que l'induction soit réglée de telle sorte que la trajectoire centrale soit confondue avec $\mathrm{x}^{\prime} \mathrm{x}$; le milieu de $\mathrm{H}_{1} \mathrm{H}_{2}$ sera alors confondu avec $\Omega$. Une trajectoire issue, par exemple, d'un point $\mathrm{A}^{\prime}$ de l'image $\mathrm{I}_{0}^{\prime}$ et qui s'appuie en $H_{1}$, reviendra après réflexion en $A^{\prime}$ en passant par $\mathrm{H}_{2}$ : elle se réfléchira sur une équipotentielle $\varphi-\varphi_{1}$. Par conséquent, il faudra faire varier $V_{\mathrm{R}}$ de $\varphi$ à $\varphi-\varphi_{1}$ pour éteindre complètement l'éclairement $\mathrm{du}$ point $\mathrm{A}^{\prime}$. On peut faire un raisonnement analogue pour le point $\mathbf{B}^{\prime}$, mais la situation est en fait un peu plus compliquée à cause des aberrations d'ouverture du prisme qui modifient la position de $C^{\prime}$ et celles du miroir qui pourraient changer la position du centre. Pour avoir une idée de l'ordre de grandeur de $\varphi_{1}$ et de la dépendance du filtrage avec $\mathbf{B}^{\prime}$, nous avons eu recours à l'expérience.

Une source thermo-ionique d'ions $\mathrm{Na}^{+}$ou $\mathrm{Cs}^{+}\left({ }^{1}\right)$ remplace l'objet. Cette source présente l'intérêt d'avoir une dispersion d'énergie relativement faible. Par un jeu de diaphragmes [7], on peut réduire les dimensions de $\mathrm{C}$ (donc celles de $\mathrm{C}^{\prime}$ ) et celles du champ imagé (donc limiter la position de $\mathbf{B}^{\prime}$ au voisinage de $\mathbf{A}^{\prime}$ ). Dans ces conditions, le prisme dirige vers le miroir un pinceau fin de trajectoires qu'on peut faire tourner autour de $\mathrm{S}$ en jouant sur l'induction magnétique. Il suffit alors de relever la courbe de contre-champ lorsque ce pinceau est confondu avec $\mathrm{x}^{\prime} \mathrm{x}$ et lorsque ce pinceau est incliné sur $\mathrm{x}^{\prime} \mathrm{x}$ pour mesurer le décalage en énergie $\varphi_{1}$ qui en résulte. Nous avons fait l'expérience en décalant le pinceau de $\pm 0,3 \mathrm{~mm}$ le long de $\mathrm{y}^{\prime} \mathrm{y}$, de part et d'autre de $\Omega$; ceci est équivalent à une dispersion de $\pm 7,5 \mathrm{eV}$ donnée par le prisme (la dispersion relevée expérimentalement le long de l'axe $\mathrm{y}^{\prime} \mathrm{y}$ vaut $40 \mu / \mathrm{V})$. Nous avons trouvé un décalage des

(1) Ces ions proviennent d'aluminosilicates déposés sur un filament de tungstène chauffé par effet Joule. courbes de contre-champ égal à $0,15 \mathrm{~V}$. Dans les conditions opératoires normales, la pupille placée en $\mathrm{C}^{\prime}$ a une forme elliptique dont les demi-axes le long de $\mathrm{y}^{\prime} \mathrm{y}$ et dans la direction perpendiculaire au plan de la figure 2, sont respectivement de l'ordre de 0,15 et $0,05 \mathrm{~mm}$. Etant donné que le miroir fonctionne comme une lentille à émission [5], il y a une relation entre $\varphi_{1}$ et l'écart à l'axe de la trajectoire au niveau de $C^{\prime}$. On peut admettre que cet écart est proportionnel à $\sqrt{\varphi_{1}}$ (ce qui est le cas pour la première lentille à émission). On voit alors que $\varphi_{1}$ est certainement inférieur à $0,05 \mathrm{~V}$.

Par ailleurs, en jouant sur le diaphragme de champ, on peut limiter un pinceau situé autour de $\mathbf{B}^{\prime}$ et vérifier que la courbe de contre-champ n'est pratiquement pas décalée par rapport à celle qu'on obtient avec le pinceau situé près de $\mathrm{A}^{\prime}$. En définitive, on peut affirmer que l'erreur absolue sur le repérage de l'énergie d'un faisceau centré est au plus de l'ordre de $0,05 \mathrm{~V}$ pour tout le champ image.

2.2.2 Faisceau d'ions présentant une dispersion énergétique. - Considérons tout d'abord un faisceau réduit, avant d'entrer dans le prisme magnétique, à sa trajectoire centrale. Après la première déflexion, les ions de différentes énergies se déploient suivant un éventail de trajectoires rectilignes qui s'appuient sur $F$. Un des rayons de l'éventail sera confondu avec $\mathrm{x}^{\prime} \mathrm{x}$ et parcouru par des ions d'énergie $q \varphi_{\mathrm{c}}$.

Supposons que la fente de sélection soit réglée de manière à isoler, autour de $\varphi_{\mathrm{c}}$, une bande d'énergie dont les bornes sont $q \varphi_{1}$ et $q \varphi_{2}\left(\varphi_{1}<\varphi_{2}\right.$ et $\left.\varphi_{\mathrm{c}}=\left(\varphi_{1}+\varphi_{2}\right) / 2\right)$. Lorsque $V_{\mathrm{R}}$ est égal à $\varphi_{\mathrm{c}}$, tous les ions d'énergie égale ou supérieure à $\varphi_{c}$ sont neutralisés. En faisant varier $V_{\mathrm{R}}$ on peut tracer une courbe de contre-champ, mais il faut remarquer que les ions d'énergie $q \varphi_{1}$ et $q \varphi_{2}$ sont complètement arrêtés pour des valeurs de $V_{\mathrm{R}}$ respectivement égales à $\varphi_{1}-\varphi_{11}$ et $\varphi_{2}-\varphi_{21}$. D'après ce que nous savons, $\varphi_{11}$ et $\varphi_{21}$ peuvent être de l'ordre de $0,15 \mathrm{~V}$ pour une bande de $\pm 7,5 \mathrm{~V}$ autour de $\varphi_{\mathrm{c}}$. Cependant, cet effet a d'autant moins d'importance que la bande est plus étroite. Ainsi, pour des ions qui correspondraient à une bande de $\pm 2,5 \mathrm{~V}$ autour de $\varphi_{\mathrm{c}}$, on peut estimer que $\varphi_{1}$ est inférieur à $0,02 \mathrm{~V}$.

Lorsqu'on considère le faisceau complet des ions, on arrive à des conclusions qui restent essentiellement les mêmes que celles relatives aux ions monocinétiques. Cependant, la situation est ici encore compliquée par les aberrations chromatiques du prisme et du miroir [7]. Pour réduire d'éventuels effets chromatiques (dont nous n'avons pas étudié en détail l'influence sur les courbes de contre-champ), nous avons pris comme règle pratique de relever les courbes de contre-champ dans un intervalle de $\pm 2,5 \mathrm{~V}$ autour de $\varphi_{\mathrm{c}}$.

2.3 OBTENTION D'UNE DISTRIBUTION ÉNERGÉTIQUE. - $\mathrm{Si} E_{\mathrm{c}}$ est l'énergie cinétique initiale des ions de charge $q$ (rappelons que $E_{\mathrm{c}}$ est mesuré en électrons- 
volts), à leur sortie du métal, et $V$ la tension appliquée à l'objet, ces ions seront réfléchis dans le miroir si $q V_{\mathrm{R}}>q V+e E_{\mathrm{c}}$. Ils seront arrêtés dès que

$$
q V_{\mathrm{R}}=q V+e E_{\mathrm{c}} .
$$

La coupure à $E_{\mathrm{c}}$ étant définie avec une incertitude qui dépend de l'inclinaison de la trajectoire des ions par rapport à l'axe du miroir et dont la valeur limite a été fixée au paragraphe précédent. La distribution en énergie des ions recueillis dérive de la courbe de contre-champ $I_{\mathrm{r}}\left(V_{\mathrm{R}}-V\right)$ relevée en faisant varier la différence de potentiel $V_{\mathrm{R}}-V$; soit :

$$
n_{\mathrm{D}}\left(E_{\mathrm{c}}\right)=\frac{e}{q^{2}} \frac{\mathrm{d} I_{\mathrm{r}}\left(V_{\mathrm{R}}-V\right)}{\mathrm{d}\left(V_{\mathrm{R}}-V\right)} .
$$

En fait, si l'on tient compte de la différence de potentiel de contact entre l'électrode répulsive et l'objet, tout se passe comme si les ions avaient une énergie initiale égale à

$$
E_{\mathrm{c}}+\frac{q}{e}\left(W_{\mathrm{M}}-W_{\mathrm{R}}\right)
$$

$W_{\mathrm{M}}$ et $W_{\mathrm{R}}$ étant les potentiels de surface de l'objet et de l'électrode $\mathrm{R}$.

On a donc une coupure à $E_{\mathrm{c}}$ lorsque l'égalité suivante est réalisée :

$$
q V_{\mathrm{R}}=q V+e E_{\mathrm{c}}+q\left(W_{\mathrm{M}}-W_{\mathrm{R}}\right) .
$$

Si l'on pose $V_{\mathrm{R}}-V=\Delta v$, on a :

$$
\Delta v=\frac{e}{q} E_{\mathrm{c}}+\left(W_{\mathrm{M}}-W_{\mathrm{R}}\right) .
$$

Par conséquent, la distribution en énergie $n_{\mathrm{D}}\left(E_{\mathrm{c}}\right)$ (3) est obtenue, dans la pratique, après avoir dérivé le courant d'ions recueillis $I_{\mathrm{r}}(\Delta v)$ par rapport à $\Delta v$ et après avoir effectué une translation de cette courbe égale à $-\left(W_{\mathrm{M}}-W_{\mathrm{R}}\right)$. On obtient finalement la distribution réelle des ions $n\left(E_{\mathrm{c}}\right)$ en appliquant la correction donnée par l'expression (2) qui tient compte de la distribution angulaire des ions suivant une loi de Lambert.

D'après (4), lorsque $\Delta v=W_{\mathrm{M}}-W_{\mathrm{R}}$, les ions d'énergie cinétique nulle sont arrêtés et par conséquent le courant $I_{\mathrm{r}}$ s'annule. On mesure alors la différence de potentiel de contact entre l'électrode $\mathrm{R}$ et l'échantillon. Cette mesure permet de remonter au travail de sortie d'un métal. Il suffit pour cela de mesurer la différence des potentiels de contact $W_{\mathbf{M}_{1}}-W_{\mathbf{M}_{2}}$ déduite de l'analyse respective des distributions énergétiques d'ions venant de cibles $M_{1}$ et $M_{2}$ et de prendre le travail de sortie d'un métal donné comme valeur de référence. Nous avons choisi la valeur de $4,55 \mathrm{eV}$ du travail de sortie du tungstène polycristallin [8] et dressé ainsi une liste des travaux de sortie [9].

Cette méthode de mesure des travaux de sortie est intéressante à deux points de vue. Elle a permis d'une part de constater que le bombardement ionique n'apportait pas de modification importante du travail de sortie d'un métal et d'autre part, elle donne une mesure in situ de l'un des paramètres qui contrôle l'émission ionique [3], [6].

Lorsqu'on veut placer le zéro d'énergie d'une distribution, on a besoin de connaître $W_{\mathrm{R}}$. Mais l'état de surface de l'électrode $R$ est mal défini à cause de l'influence du gaz résiduel et de l'implantation d'ions de nature différente, et par conséquent, $W_{R}$ l'est également. Pour réduire ces effets nous avons continuellement chauffé l'électrode $\mathrm{R}$ au cours de nos expériences. Dans ces conditions, un examen de la position relative des courbes de contre-champ obtenues pour des ions positifs et négatifs émis par un même échantillon [9] permet d'estimer une incertitude de $0,1 \mathrm{~V}$ sur le repérage absolu du zéro.

3. Distributions énergétiques des ions émis par les métaux purs. - 3.1 DisTRIBUTIONS ÉNERGÉTIQUES DES IONS SIMPLES. - Les distributions des ions $\mathrm{Cu}^{+}$et $\mathrm{Ni}^{+}$sont présentées (Fig. 3 et 4 ) avant et après la correction de Lambert. Cette correction tend à restituer dans son intégralité l'émission ionique d'un élément. Elle est importante au-delà de quelques électrons-volts, mais on remarquera qu'elle ne change pas les caractéristiques relatives des distributions, car le rapport du nombre d'ions $\mathrm{Cu}^{+}$au nombre d'ions $\mathrm{Ni}^{+}$, à une énergie donnée, est conservé. Elle permet, néanmoins, de préciser certains points, comme par exemple, l'énergie la plus probable.

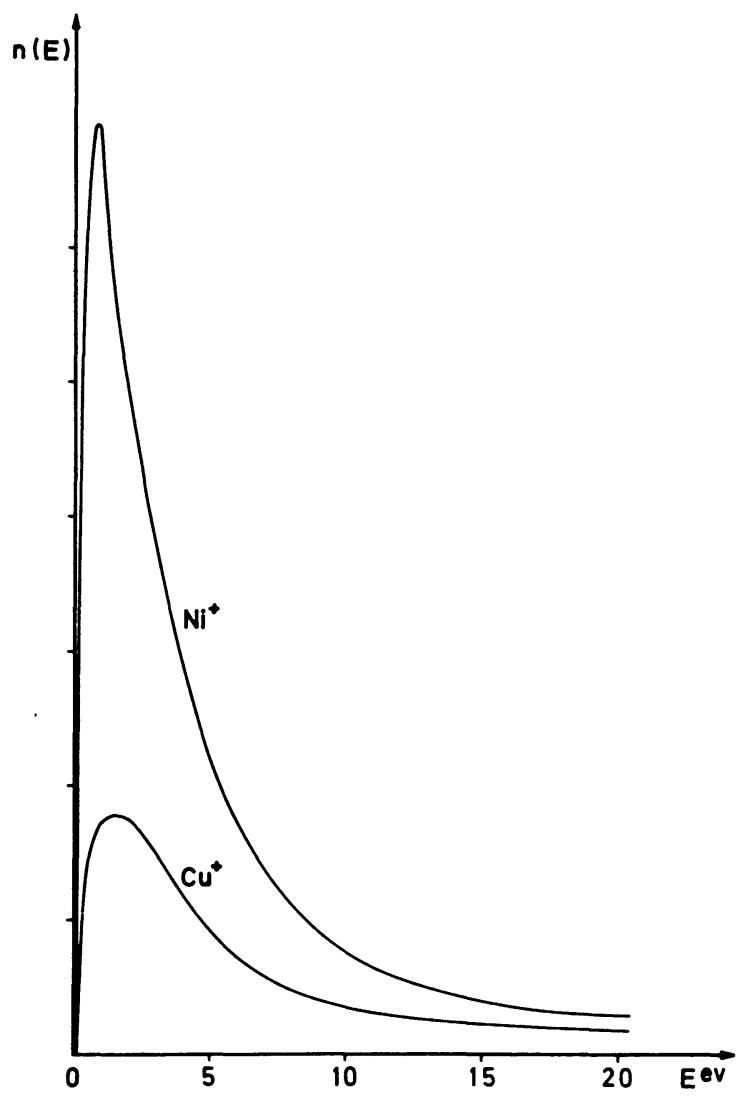

Fig. 3. - Distributions énergétiques en vraies grandeurs des ions $\mathrm{Cu}^{+}$et $\mathrm{Ni}^{+}$avant la correction de Lambert. 


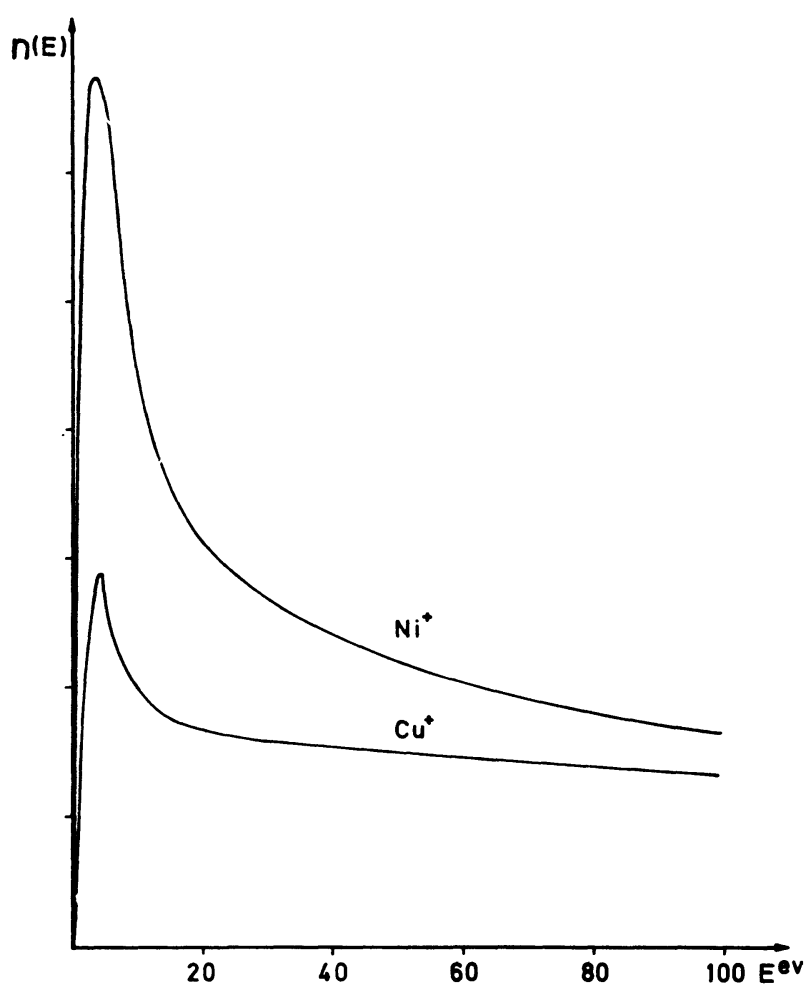

Fig. 4. - Distributions énergétiques en vraies grandeurs des ions $\mathrm{Cu}^{+}$et $\mathrm{Ni}^{+}$après la correction de Lambert.

Les caractéristiques essentielles des distributions énergétiques des ions $\mathrm{Cu}^{+}$et $\mathrm{Ni}^{+}$(Fig. 4) sont les suivantes :

- l'émission ionique du cuivre, comparée à celle du nickel, présente un déficit d'ions de basse énergie,

- l'énergie la plus probable de la distribution des ions $\mathrm{Cu}^{+}$est de l'ordre de $4,5 \mathrm{eV}$, alors que celle de la distribution des ions $\mathrm{Ni}^{+}$est un peu plus faible, de l'ordre de $3,5 \mathrm{eV}$,

- le rapport du nombre d'ions $\mathrm{Cu}^{+}$au nombre d'ions $\mathrm{Ni}^{+}$, pris à même énergie $E_{\mathrm{c}}, n_{\mathrm{i}}\left(\mathrm{Cu}^{+}\right) / n_{\mathrm{i}}\left(\mathrm{Ni}^{+}\right)$, croît régulièrement à mesure que $E_{\mathrm{c}}$ augmente jusqu'à atteindre une valeur à peu près constante au-delà de $30 \mathrm{eV}$. Quand on cherche à préciser ce point, on remarque que $\log n_{\mathrm{i}}\left(\mathrm{Cu}^{+}\right) / n_{\mathbf{i}}\left(\mathrm{Ni}^{+}\right)$varie à peu près linéairement en fonction de l'inverse de la vitesse $v$ d'éjection des ions (Fig. 5). C'est aux faibles vitesses d'éjection, correspondant à des énergies maximales de

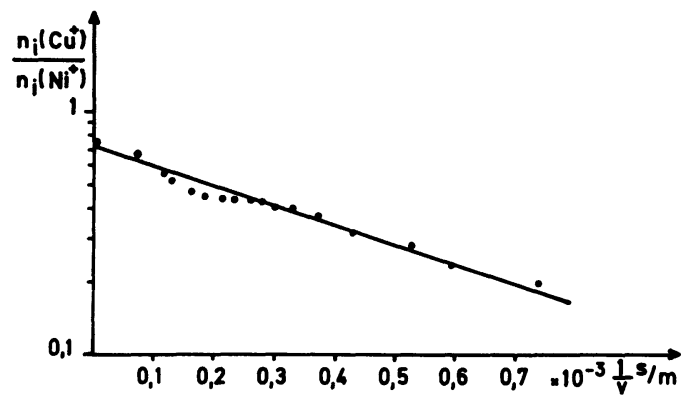

Fig. 5. - Rapport $n_{\mathrm{i}}\left(\mathrm{Cu}^{+}\right) / \boldsymbol{n}_{\mathrm{i}}\left(\mathrm{Ni}^{+}\right)$en fonction de l'inverse de la vitesse d'éjection des ions.
4 à $5 \mathrm{eV}$, que les points sont les mieux alignés sur la figure 5. Au-delà de ces valeurs, on observe un écart à la linéarité de l'ordre de $10 \%$ au maximum (cette dispersion n'excède pas la précision des mesures). On a donc la relation :

$$
\frac{n_{\mathrm{i}}\left(\mathrm{Cu}^{+}\right)}{n_{\mathrm{i}}\left(\mathrm{Ni}^{+}\right)}=W_{0} \exp -\frac{v_{0}}{v}
$$

avec les valeurs expérimentales suivantes :

$$
v_{0}=1,7 \times 10^{3} \mathrm{~m} / \mathrm{s} \quad \text { et } \quad W_{0}=0,72 .
$$

L'expression (5) tend rapidement vers $W_{0}$ lorsque l'énergie cinétique des ions augmente et au-delà de $30 \mathrm{eV}$ environ, on peut pratiquement considérer que les distributions énergétiques des ions $\mathrm{Cu}^{+}$et $\mathrm{Ni}^{+}$ sont homothétiques (Fig. 4).

Nous avons étudié les distributions énergétiques des ions simples $\mathrm{M}^{+}$émis par les métaux de la série $3 \mathrm{~d}$. Pour établir un premier classement, il suffit de comparer les rendements $\mathrm{K}\left(\mathrm{M}^{+}\right)$(c'est-à-dire le nombre d'ions secondaires $\mathbf{M}^{+}$éjectés par ion incident) mesurés pour différentes valeurs de l'énergie $E_{\mathrm{c}}$ des ions $\mathbf{M}^{+}$. Nous avons choisi de mesurer les rendements $\mathrm{K}^{0}\left(\mathrm{M}^{+}\right)$en ions d'énergie comprise entre 0 et $15 \mathrm{eV}$ et les rendements $\mathrm{K}^{50}\left(\mathrm{M}^{+}\right)$et $\mathrm{K}^{100}\left(\mathrm{M}^{+}\right)$ pour lesquels l'énergie est comprise dans une bande de $\pm 7,5 \mathrm{eV}$ de largeur, respectivement autour de 50 et $100 \mathrm{eV}\left(^{2}\right)$ (les rendements $\mathrm{K}^{0}\left(\mathrm{M}^{+}\right)$ont également été mesurés pour des éléments d'autres séries de transition (Fig. 6)).

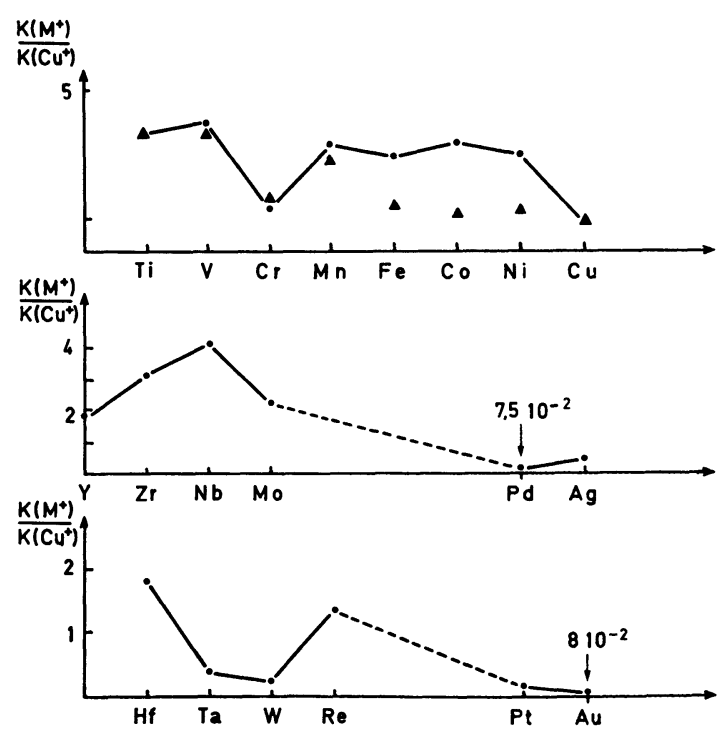

FIG. 6. - Rendements ioniques des éléments de transition

- Rendements $\mathrm{K}^{0}\left(\mathrm{M}^{+}\right) / \mathrm{K}^{0}\left(\mathrm{Cu}^{+}\right)$.

$\triangle$ Rendements $\mathrm{K}^{100}\left(\mathrm{M}^{+}\right) / \mathrm{K}^{100}\left(\mathrm{Cu}^{+}\right)$.

(2) La largeur de la bande d'énergie est naturellement fixée par la largeur de la fente de sélection. Sur le tableau I et la figure 6 , nous avons porté les valeurs $\mathrm{K}\left(\mathrm{M}^{+}\right)$normalisées au cuivre. 
TABLEAU I

\begin{tabular}{|c|c|c|c|c|c|c|c|c|}
\hline & $\mathrm{Ti}$ & $\mathrm{V}$ & $\mathrm{Cr}$ & $\mathrm{Mn}$ & $\mathrm{Fe}$ & Co & $\mathrm{Ni}$ & $\mathrm{Cu}$ \\
\hline & - & - & - & - & - & - & - & - \\
\hline$\frac{\mathrm{K}\left(\mathrm{M}^{+}\right)}{\mathrm{K}^{0}\left(\mathrm{Cu}^{+}\right)}$ & 3,55 & 4,1 & 1,3 & 3,3 & 3 & 3,4 & 3 & 1 \\
\hline$\frac{\mathrm{K}^{50}\left(\mathrm{M}^{+}\right)}{\mathrm{K}^{50}\left(\mathrm{Cu}^{+}\right)}$ & 3,9 & 4 & 2 & 2,9 & 1,75 & 1,95 & 1,48 & 1 \\
\hline$\frac{\mathrm{K}^{100}\left(\mathrm{M}^{+}\right)}{\mathrm{K}^{100}\left(\mathrm{Cu}^{+}\right)}$ & 3,4 & 3,5 & 1,5 & 2,8 & 1,4 & 1,4 & 1,25 & 1 \\
\hline$\frac{\mathrm{K}\left(\mathrm{M}^{+}\right)}{\mathrm{K}\left(\mathrm{Cu}^{+}\right)}$global & 2,7 & & & & 1,7 & & 1,33 & 1 \\
\hline$\frac{r_{\mathrm{M}}}{r_{\mathrm{Cu}}}=\frac{\mathrm{K}^{0}\left(\mathrm{M}^{+}\right) / \mathrm{K}^{0}\left(\mathrm{Cu}^{+}\right)}{\mathrm{K}^{100}\left(\mathrm{M}^{+}\right) / \mathrm{K}^{100}\left(\mathrm{Cu}^{+}\right)}$ & 1,05 & 1,17 & 0,87 & 1,18 & 2,14 & 2,42 & 2,4 & 1 \\
\hline
\end{tabular}

Le tableau I montre que pour la série $3 \mathrm{~d}$, les rendements relatifs, normalisés par rapport au cuivre, ne varient pratiquement plus en fonction de l'énergie des ions, au-delà d'une trentaine d'électrons-volts. On voit également que le groupe des ferromagnétiques $(\mathrm{Fe}, \mathrm{Co}, \mathrm{Ni})$ est caractérisé par des valeurs

$$
\frac{\mathrm{K}^{0}\left(\mathrm{M}^{+}\right)}{\mathrm{K}^{0}\left(\mathrm{Cu}^{+}\right)}
$$

plus élevées que les valeurs des rendements

$$
\frac{\mathrm{K}^{50}\left(\mathrm{M}^{+}\right)}{\mathrm{K}^{50}\left(\mathrm{Cu}^{+}\right)} \quad \text { et } \quad \frac{\mathrm{K}^{100}\left(\mathrm{M}^{+}\right)}{\mathrm{K}^{100}\left(\mathrm{Cu}^{+}\right)}
$$

De cet ensemble de résultats, on peut en conclure qu'au-delà de $30 \mathrm{eV}$, les distributions énergétiques de tous les ions de la série sont homothétiques. Par contre, à mesure que l'énergie des ions diminue, une analyse plus fine montre que les distributions énergétiques se différencient. C'est cette évolution que reflètent les rendements relatifs indiqués figure 6 et tableau $I$. L'effet n'est particulièrement sensible qu'en dessous d'une quinzaine d'électrons-volts.

Il est commode de normaliser toutes les émissions à haute énergie, $100 \mathrm{eV}$ par exemple, de manière à montrer les différences qui se produisent en dessous de $15 \mathrm{eV}$, dans les distributions énergétiques. On définit alors pour chaque élément $M$ un rendement normalisé de basse énergie $r_{\mathrm{M}}=\mathrm{K}^{0}\left(\mathrm{M}^{+}\right) / \mathrm{K}^{100}\left(\mathrm{M}^{+}\right)$et l'on compare entre eux les rapports $r_{\mathrm{M}} / r_{\mathrm{Cu}}$ (tableau I). $r_{\mathrm{M}} / r_{\mathrm{Cu}}$ mesure, en quelque sorte, l'écart à une distribution du type de celle des ions cuivre. Lorsqu'on remonte la série $3 \mathrm{~d}$, à partir du cuivre, on trouve successivement (Fig. 7) :

$1^{\text {er }}$ groupe de métaux : le cuivre seul avec $r_{\mathrm{Cu}} / r_{\mathrm{Cu}}=1$.

$2^{e}$ groupe de métaux: les ferromagnétiques $(\mathrm{Ni}$, $\mathrm{Co}, \mathrm{Fe})$ avec $r_{\mathrm{M}} / r_{\mathrm{Cu}} \sim 2,3$.

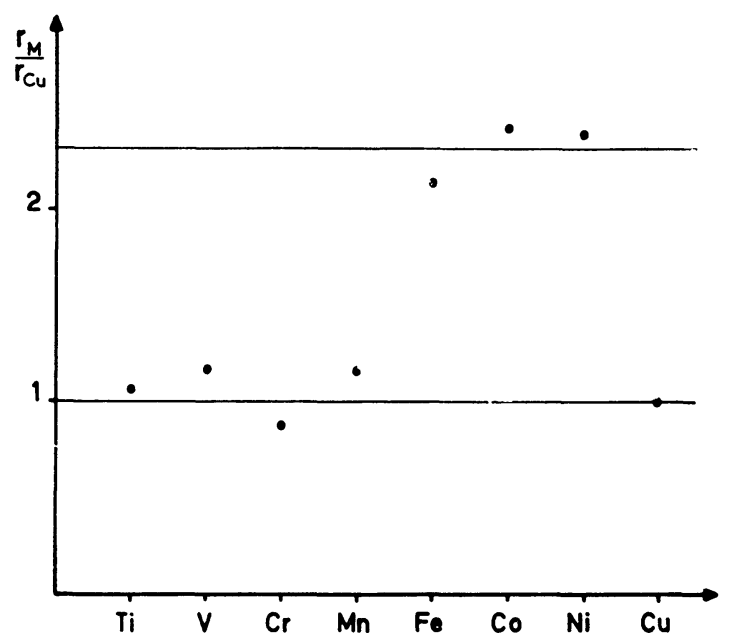

FIG. 7. - Rendements normalisés de basse énergie

$$
\begin{gathered}
\frac{r_{\mathrm{M}}}{r_{\mathrm{Cu}}}=\frac{\mathrm{K}^{0}\left(\mathrm{M}^{+}\right) / \mathrm{K}^{0}\left(\mathrm{Cu}^{+}\right)}{\mathrm{K}^{100}\left(\mathrm{M}^{+}\right) / \mathrm{K}^{100}\left(\mathrm{Cu}^{+}\right)} \\
\text {des éléments de transition. }
\end{gathered}
$$

$3^{\mathrm{e}}$ groupe de métaux : $\mathrm{Mn}, \mathrm{Cr}, \mathrm{V}$, Ti pour lesquels $r_{\mathrm{M}} / r_{\mathrm{Cu}} \sim 1$.

Les distributions énergétiques obtenues avec le miroir électrostatique autorisent une comparaison beaucoup plus détaillée des rendements $\mathrm{K}\left(\mathrm{M}^{+}\right)$mesurés en fonction de l'énergie $E_{\mathrm{c}}$. Leur examen confirme les conclusions précédentes.

Sur la figure 8, on a reporté les distributions des ions $\mathrm{Cu}^{+}, \mathrm{Ni}^{+}$et $\mathrm{Cr}^{+}$(les trois éléments choisis correspondent respectivement aux trois groupes indiqués précédemment). Les distributions ont été normalisées à $100 \mathrm{eV}$ de manière à faire ressortir les différences qui apparaissent à basse énergie. On constate, en particulier, la grande ressemblance entre les spectres d'énergie des ions $\mathrm{Cr}^{+}$et $\mathrm{Cu}^{+}$.

Le rendement ionique de basse énergie, inférieure à $30 \mathrm{eV}$, ne représente qu'une faible part, estimée au 
maximum à $20 \%$, de l'émission globale d'un élément. Il n'est donc pas étonnant que l'énergie moyenne des ions soit sensiblement la même pour tous les éléments de la série (20) et que les rendements ioniques globaux qui sont portés sur le tableau I soient porportionnels aux rendements partiels $\mathrm{K}^{50}\left(\mathrm{M}^{+}\right)$et $\mathrm{K}^{100}\left(\mathrm{M}^{+}\right)$, mesurés à haute énergie.

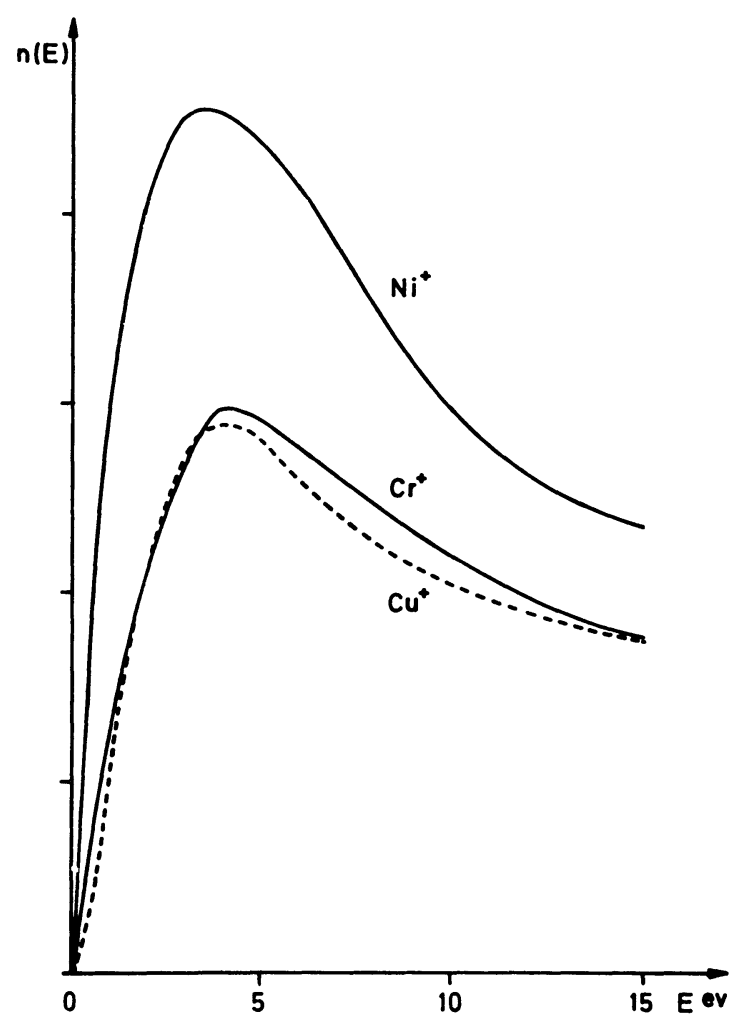

Fig. 8. - Distributions énergétiques des ions $\mathrm{Cu}^{+}, \mathrm{Cr}^{+}$et $\mathrm{Ni}^{+}$. Ces courbes ont été normalisées à $100 \mathrm{eV}$.

La plupart des expérimentateurs qui ont proposé une interprétation empirique de l'émission ionique de cibles bombardées par des ions de gaz rare de quelques $\mathrm{keV}$ d'énergie [10], [11], se sont référés à des mesures de rendements en ions lents. Or, si l'on admet que les ions secondaires proviennent en majeure partie de l'ionisation de particules neutres erratiques [12] et que les rendements et les distributions énergétiques de ces particules sont voisins pour les métaux de la série $3 \mathrm{~d}$, nos résultats expérimentaux suggèrent qu'un processus d'ionisation devrait, en premier lieu, rendre compte de l'émission des ions rapides [3]. Les caractéristiques particulières de l'émission des ions lents pourraient alors simplement traduire l'existence de processus qui interviendraient à la surface de la cible pour limiter plus ou moins la production des ions de faible énergie. En effet, les spectres d'énergie des particules neutres de cuivre et de nickel ne présentent pas en dessous de $15 \mathrm{eV}$ de différence notable [13]. C'est, par conséquent, à l'efficacité des processus d'ionisation qu'il faut attribuer les différences relevées entre les spectres d'énergie des ions émis par ces deux éléments.

La dépendance en vitesse de l'expression (5) qui relie les spectres des ions $\mathrm{Cu}^{+}$et $\mathrm{Ni}^{+}$suggère que la production des ions secondaires est en partie limitée par des mécanismes d'échange électronique entre le métal et l'atome. Nous montrerons dans un prochain article que cette interprétation entre dans le cadre d'une analyse des processus destructifs des états autoionisants $\left({ }^{3}\right)$. Processus qui conduiraient à une perte d'ions $\mathrm{Cu}^{+}$plus importante que la perte des ions $\mathrm{Ni}^{+}$.

En ce qui concerne les éléments légers, aluminium et magnésium, nous observons des distributions énergétiques de formes assez semblables (Fig. 9). Ce résultat ne concorde pas avec ce qu'Hennequin [2] signale par ailleurs pour l'émission de ces éléments, à savoir une énergie moyenne des ions $\mathrm{Mg}^{+}$beaucoup plus forte que celle des ions $\mathrm{Al}^{+}$. Cela aurait dû normalement déplacer la distribution des ions $\mathrm{Al}^{+}$par rapport à celle des ions $\mathrm{Mg}^{+}$vers les très basses énergies, ce qui ne semble pas se produire.

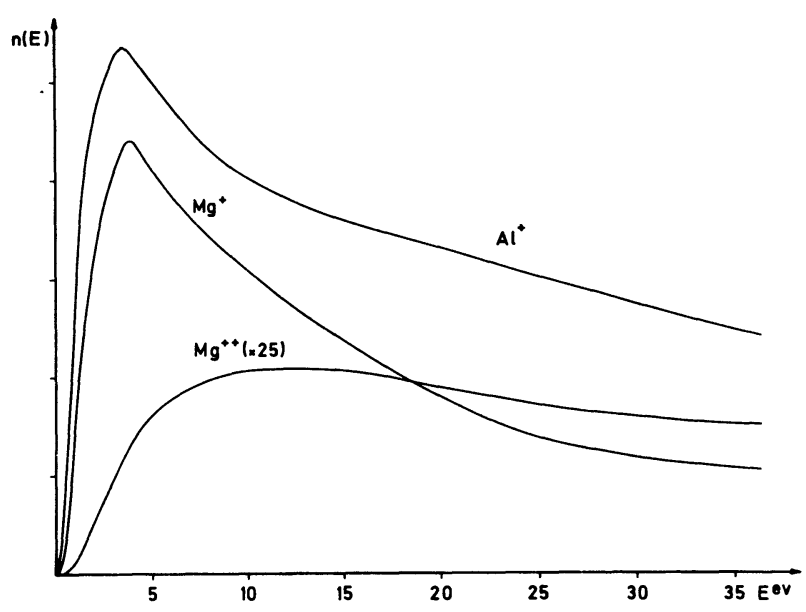

FIG. 9. - Distributions énergétiques en vraies grandeurs des ions $\mathrm{Al}^{+}, \mathrm{Mg}^{+}$et $\mathrm{Mg}^{++}$.

3.2 Distributions ÉNERGÉTIQUES DES IONS MOLÉCulaires. - Les distributions des ions $\mathrm{Cu}_{2}^{+}, \mathrm{Cu}_{3}^{+}$, $\mathrm{Ni}_{2}^{+}$et $\mathrm{Ni}_{3}^{+}$et $\mathrm{Al}_{2}^{+}, \mathrm{Al}_{3}^{+}$sont présentées figures $10,11,12$. D'une manière générale, la dispersion énergétique des ions moléculaires est beaucoup plus étroite que celle des ions simples : l'émission au-delà de $15 \mathrm{eV}$ devient extrêmement faible. Toutefois, ces distributions ne sont pas tassées vers les très basses énergies. La croissance jusqu'au maximum est plus lente que pour les ions simples et les énergies les plus probables, ellesmêmes, se situent à plusieurs eV.

(3) Rappelons que le modèle développé pour expliquer l'émission ionique des métaux de transition repose sur la formation d'états auto-ionisants [3]. 


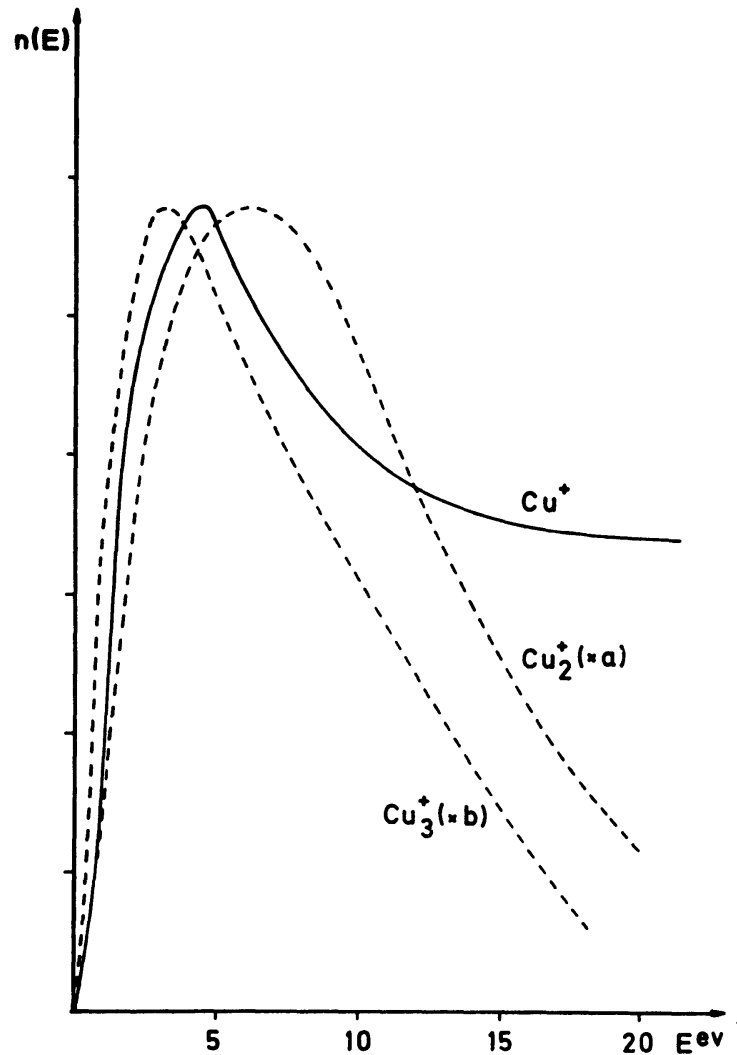

FIG. 10. - Distributions énergétiques des ions $\mathrm{Cu}^{+}, \mathrm{Cu}_{2}^{+}$et $\mathrm{Cu}_{3}^{+}$ normalisées au maximum : $a=3,6 ; b=2,1$.

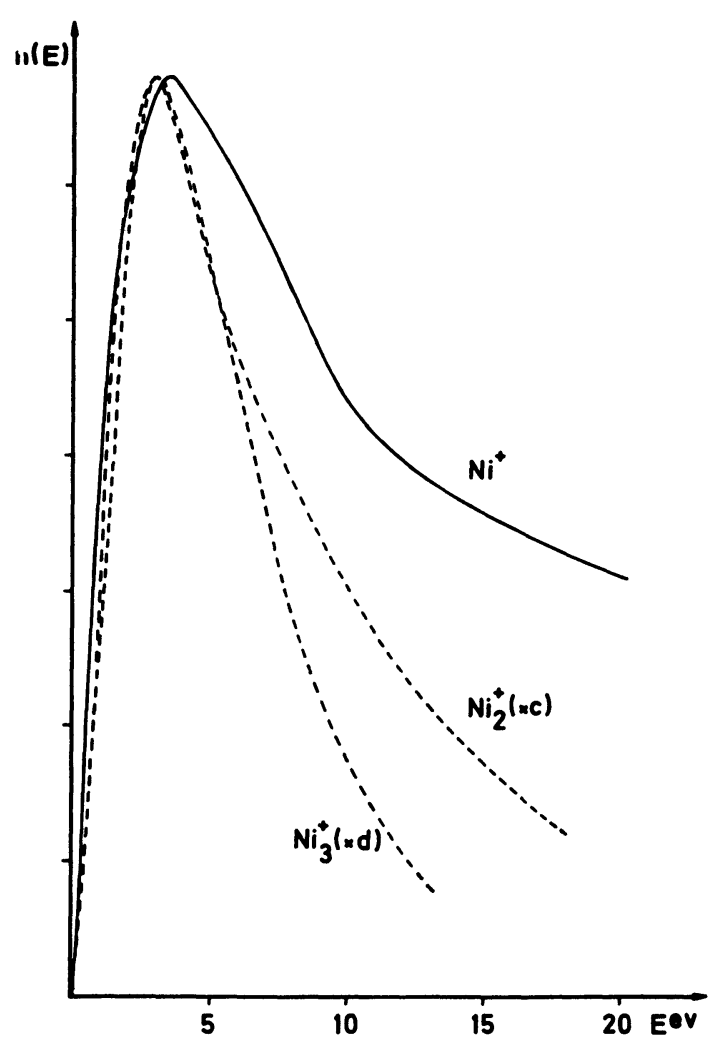

Fig. 11. - Distribution énergétique des ions $\mathrm{Ni}^{+}, \mathrm{Ni}_{2}^{+}$et $\mathrm{Ni}_{3}^{+}$ normalisées au maximum : $c=2,7 ; d=24,2$.

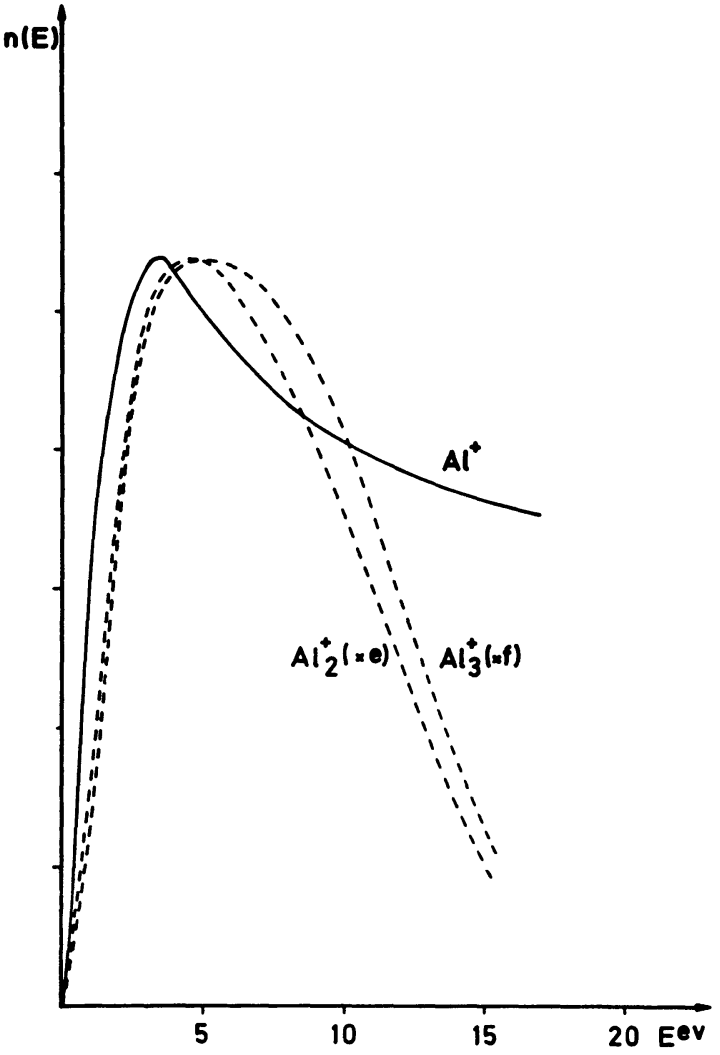

FIG. 12. - Distributions énergétiques des ions $\mathrm{Al}^{+}, \mathrm{Al}_{2}^{+}$et $\mathrm{Al}_{3}^{+}$ normalisées au maximum : $e=3 ; f=11,5$.

On note un aspect particulier de la distribution de chacune des espèces étudiées. Ainsi, les ions $\mathrm{Al}_{2}^{+}$ et $\mathrm{Al}_{3}^{+}$présentent des spectres très arrondis où les maximums se situent respectivement vers 4,5 et $5,5 \mathrm{eV}$, alors que le maximum de la distribution des ions simples $\mathrm{Al}^{+}$est à $3,5 \mathrm{eV}$. Au contraire, les distributions des ions $\mathrm{Ni}_{2}^{+}$et $\mathrm{Ni}_{3}^{+}$sont assez pointues avec une énergie la plus probable inférieure à celle des ions simples. Les spectres des ions $\mathrm{Cu}_{3}^{+}$et $\mathrm{Ni}_{3}^{+}$sont tout à fait semblables, par contre ceux des ions $\mathrm{Cu}_{2}^{+}$et $\mathrm{Ni}_{2}^{+}$ sont totalement différents. Il y a un appauvrissement manifeste en ions $\mathrm{Cu}_{2}^{+}$de basse énergie qui se traduit par une distribution très arrondie, un maximum à une énergie élevée de $6 \mathrm{eV}$ et une intensité plus faible que celle des ions $\mathrm{Cu}_{3}^{+}$. Au-delà de $15 \mathrm{eV}$, il semble cependant que la situation redevienne identique à celle du nickel puisque le nombre d'ions $\mathrm{Cu}_{2}^{+}$est alors supérieur au nombre d'ions $\mathrm{Cu}_{3}^{+}$.

La forme même de ces distributions énergétiques montre qu'un mécanisme d'évaporation thermique n'est probablement pas à l'origine de la formation de ces groupements moléculaires. On imaginerait plutôt un processus cinétique où, par exemple, plusieurs atomes voisins sur la surface du métal, sont mis presque simultanément en mouvement par l'impact d'une ou plusieurs particules déplacées à la suite de la cascade de collisions initiée par l'arrivée d'un ion primaire. 
Il est en fait difficile de communiquer une énergie cinétique importante à un groupement polyatomique sans le dissocier. Les distributions énergétiques des figures 10,11 et 12 sont très significatives à cet égard.

Toutefois, le comportement des ions $\mathrm{Cu}_{2}^{+}$et $\mathrm{Cu}_{3}^{+}$est curieux. En effet, l'énergie de dissociation de l'ion $\mathrm{Cu}_{2}^{+}$ est plus faible que celle de $\mathrm{Cu}_{3}^{+}$[14], néanmoins, au-dessus de $15 \mathrm{eV}$, on rencontre davantage d'ions $\mathrm{Cu}_{2}^{+}$. Cette situation traduit vraisemblablement le fait qu'il devient de plus en plus difficile de communiquer une énergie cinétique importante lorsque le nombre d'atomes croît mais qu'en revanche, l'énergie d'excitation emportée par un paquet d'atomes peut alors devenir de plus en plus élevée. La désexcitation du groupement peut ensuite conduire à son ionisation à condition, entre autres, que l'énergie d'excitation emmagasinée, à la sortie, soit compatible avec la stabilité de l'édifice. On comprend que le nombre d'ions $\mathrm{Cu}_{3}^{+}$décroisse avec l'énergie beaucoup plus rapidement que celui des ions $\mathrm{Cu}_{2}^{+}$. Ce qui reste cependant inattendu c'est l'affaiblissement relatif des ions $\mathrm{Cu}_{2}^{+}$à basse énergie alors que, par ailleurs, les formes des distributions des ions $\mathrm{Ni}_{2}^{+}$et $\mathrm{Ni}_{3}^{+}$sont pratiquement identiques à basse énergie et ne se différencient nettement qu'à partir de $5 \mathrm{eV}$. Ce phénomène est peut-être lié à l'existence d'états auto-ionisants à longue durée de vie [15] et à leur "survie", jusqu'au moment où cesse l'échange électronique avec la surface, en fonction de la vitesse d'éjection.

Par ailleurs, on peut remarquer que le taux d'ionisation d'espèces telles que $\mathrm{Cu}_{n}$ doit nécessairement croître rapidement avec $n$. S'il en était autrement, il faudrait admettre qu'une fraction appréciable de la pulvérisation se fait sous forme de particules polyatomiques. On ne possède pas de données sur ce point mais il paraît peu vraisemblable qu'un rendement de pulvérisation élevé en particules $\mathrm{Cu}_{3}$, par exemple, soit passé inaperçu [16].

3.3 Distributions ÉNeRgétiQues DES IONS POLYCHARGÉs. - La distribution des ions $\mathrm{Mg}^{++}$est présentée figure 9. Elle est caractérisée par un maximum à peine prononcé, rejeté vers les hautes énergies, à $10 \mathrm{eV}$ environ, et suivi d'une lente décroissance. Il en résulte donc une énergie moyenne élevée (2). Ces constatations concernent également les émissions des ions polychargés d'aluminium (émissions qui feront l'objet d'un article ultérieur).

4. Distributions énergétiques des éléments de transition en solution dans les alliages. - On définit la constante ionique $k_{\mathrm{AB}}$ d'un alliage binaire $\mathrm{A}_{1-\mathrm{C}} \mathrm{B}_{\mathrm{C}}$ par:

$$
\frac{\mathrm{K}_{\mathrm{C}}\left(\mathrm{B}^{+}\right)}{\mathrm{K}_{1-\mathrm{C}}\left(\mathrm{A}^{+}\right)}=k_{\mathrm{AB}} \frac{\mathrm{C}}{1-\mathrm{C}}
$$

$\mathrm{K}_{1-\mathrm{c}}\left(\mathrm{A}^{+}\right)$et $\mathrm{K}_{\mathrm{C}}\left(\mathrm{B}^{+}\right)$sont les rendements ioniques des éléments $A$ et $B$ de l'alliage, de concentrations respectives $1-\mathrm{C}$ et $\mathrm{C}$.
Lorsque la concentration $\mathrm{C}$ est suffisamment faible pour que les atomes B n'interagissent pas entre eux, $k_{\mathrm{AB}}$ est indépendant de cette concentration. C'est ce que nous avons vérifié pour tous les alliages dilués où la concentration du soluté n'excédait pas $3 \%$ atomique.

Dans les alliages binaires entre métaux de transition que nous avons étudiés, les deux composants occupent des places voisines dans la classification périodique. On peut donc admettre que chaque atome d'impureté se comporte vis-à-vis des processus de collision comme un atome de la matrice. Cela revient à supposer que l'impureté participe pour une part proportionnelle à sa concentration, au rendement de pulvérisation, que ce soit sous forme d'émission erratique ou d'émission par focussons; le rendement de pulvérisation étant lui-même voisin de celui du métal pur de base. On admet également que la distribution énergétique des particules neutres de l'impureté est identique à celle de la matrice.

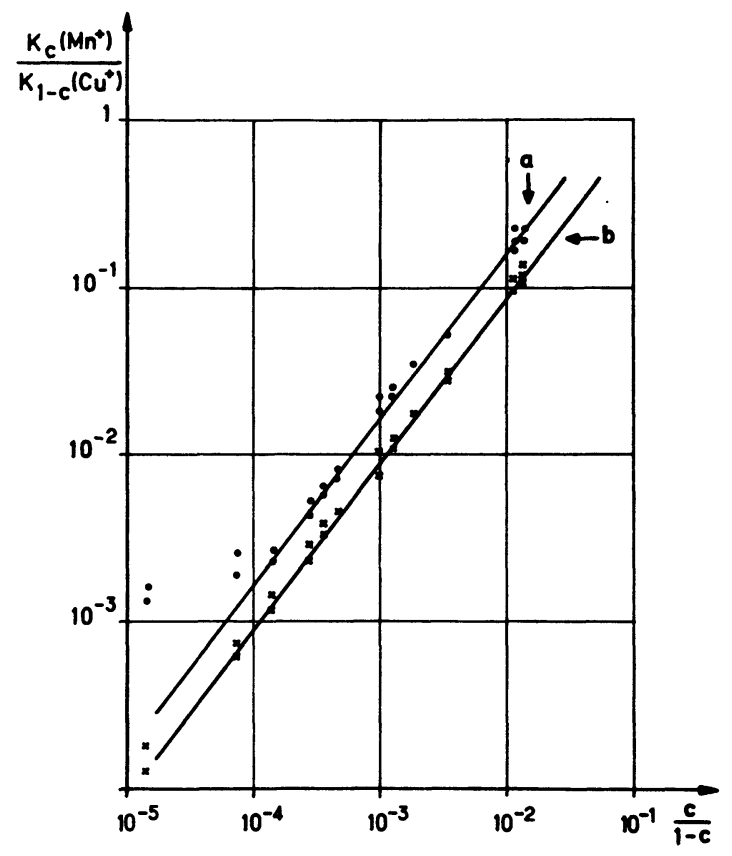

FIG. 13. - Rapport des émissions du manganèse au cuivre $\mathbf{K}\left(\mathrm{M}^{+}\right) / \mathbf{K}\left(\mathrm{Cu}^{+}\right)$dans les alliages $\mathrm{CuMn}$ en fonction du rapport des concentrations.
a) $\frac{\mathrm{K}^{0}\left(\mathrm{Mn}^{+}\right)}{\mathrm{K}^{0}\left(\mathrm{Cu}^{+}\right)}$
(ions de 0 à $15 \mathrm{eV}$ );
b) $\frac{\mathrm{K}^{E}\left(\mathrm{Mn}^{+}\right)}{\mathrm{K}^{E}\left(\mathrm{Cu}^{+}\right)}$
(ions d'énergie supérieure à $30 \mathrm{eV}$ ) .

Les alliages cuivre-manganèse ont été étudiés dans une gamme allant de $15 \mathrm{ppm}$ à $1,5 \%$ atomique. Le rapport des émissions du manganèse au cuivre $\mathrm{K}_{\mathrm{c}}\left(\mathrm{Mn}^{+}\right) / \mathrm{K}_{1-\mathrm{c}}\left(\mathrm{Cu}^{+}\right)$est porté en échelle logarithmique sur la figure 13 en fonction du rapport des concentrations $\mathrm{C} / 1-\mathrm{C}$. Cette émission a été mesurée pour les ions lents, d'énergie comprise entre 0 et 
$15 \mathrm{eV}$, et pour les ions rapides d'énergie supérieure à $30 \mathrm{eV}$ (courbes $a$ et $b$ ). La plupart des points expérimentaux se placent suivant deux droites inclinées à $45^{\circ}$, avec une dispersion inférieure à $\pm 10 \%$. Cependant, on remarque que l'intensité de l'émission des ions lents ne dépend pratiquement plus de la concentration en dessous de $100 \mathrm{ppm}$. Au contraire, pour ces faibles concentrations, les points expérimentaux se placent toujours sur la droite qui représente l'émission des ions rapides. Le fait que l'intensité du courant d'ions lents ne dépende plus de la concentration est dû à la superposition au pic de masse 55 du manganèse, d'un pic d'ions parasites de même masse. Cet effet provient vraisemblablement de la pollution de la surface de l'échantillon par des ions du type $\mathrm{AlSi}^{+}$ou $\mathrm{C}_{4} \mathrm{H}_{7}^{+}$. Cet effet de pollution de la surface de l'échantillon peut être mis en évidence facilement avec un spectrographe de masse dont la résolution est suffisante [17] $\left({ }^{4}\right)$.

La dispersion énergétique de ces pics parasites, composés d'ions moléculaires est certainement très faible, comme le montrent les distributions énergétiques. Par conséquent, l'émission ionique de haute énergie de l'impureté n'est pas affectée par leur présence. Cela explique la décroissance régulière de l'intensité en fonction de la concentration sur la courbe $b$. Il y a donc toujours moyen d'analyser un élément de faible teneur, en sélectionnant l'émission des ions rapides mais cela se fait au prix d'un abaissement de la sensibilité.

L'alignement des points expérimentaux sur la figure 13 indique bien que la constante ionique $k_{\mathrm{CuMn}}$ de l'alliage ne dépend pas de la concentration. Par contre, sa valeur varie en fonction de la bande d'énergie sélectionnée pour la mesure, puisque les droites $a$ et $b$ ne coïncident pas. On a vérifié toutefois qu'à partir de $30 \mathrm{eV}$ environ, les points expérimentaux s'alignent toujours suivant la droite $b$. Il faut donc en conclure que les distributions énergétiques des ions $\mathrm{Cu}^{+}$ et $\mathrm{Mn}^{+}$de l'alliage $\mathrm{CuMn}$ ne sont pas homothétiques en dessous de $30 \mathrm{eV}$. Il est alors intéressant de comparer les spectres d'énergie des ions émis par les alliages à ceux donnés par les métaux purs. Dans le cas présent, où l'alliage est très dilué, l'émission des ions $\mathrm{Cu}^{+}$ reste inchangée et il n'y a que les ions $\mathrm{Mn}^{+}$de l'impureté qui peuvent, éventuellement, présenter une distribution différente de celle des ions $\mathrm{Mn}^{+}$du manganèse

(4) Il ne suffit donc pas d'annoncer la limite de sensibilité d'un appareil, utilisant l'émission ionique comme moyen d'analyse, à partir du seul examen d'une impureté de concentration connue, mais très faible. Il faut encore s'assurer de la variation de cette émission en fonction de la concentration pour être sûr qu'il ne s'agit pas en fait de l'émission due à une pollution. Il ne semble pourtant pas que cette précaution ait été prise par tous les auteurs. Ce sont également des effets parasites de cette nature qui entachent vraisemblablement d'erreurs importantes les mesures de rendements ioniques négatifs des métaux purs [9]. pur. Pour mettre cela en évidence, on compare l'émission normalisée de basse énergie

$$
r_{\mathrm{Mn}}^{a}=\frac{\mathrm{K}_{\mathrm{C}}^{0}\left(\mathrm{Mn}^{+}\right)}{\mathrm{K}_{\mathrm{C}}^{100}\left(\mathrm{Mn}^{+}\right)}
$$

de l'alliage à celle

$$
r_{\mathrm{Mn}}=\frac{\mathrm{K}^{0}\left(\mathrm{Mn}^{+}\right)}{\mathrm{K}^{100}\left(\mathrm{Mn}^{+}\right)}
$$

du métal manganèse pur: soit

$$
r_{\mathrm{Mn}}^{a}=\frac{k_{\mathrm{CuMn}}^{0}}{k_{\mathrm{CuMn}}^{100}} r_{\mathrm{Cu}}
$$

où $k_{\mathrm{C} \text { Mn }}^{0}$ et $k_{\mathrm{CuMn}}^{100}$ sont les constantes ioniques déduites des droites $a$ et $b$. On trouve alors $r_{\mathrm{Mn}}^{a} / r_{\mathrm{Cu}} \sim 2$. Or, pour l'émission de basse énergie du manganèse pur (Fig. 7) on avait $r_{\mathrm{Mn}} / r_{\mathrm{Cu}} \sim 1$. Par conséquent, l'émission de basse énergie du manganèse, dans l'alliage, est tout à fait différente de celle du manganèse pur et se rapproche de l'émission des métaux ferromagnétiques pour lesquels $r_{\mathrm{Mn}} / r_{\mathrm{Cu}} \sim 2$, 3. Nous verrons par la suite d'autres cas semblables.

L'analyse quantitative d'un alliage CuMn peut se faire à partir de la mesure de l'émission des ions lents ou rapides, c'est-à-dire en utilisant l'une ou l'autre des courbes $a$ et $b$ de la figure 13. Cependant, il faut bien souligner que la mesure de l'émission de basse énergie (courbe $a$ ) est moins sûre, car elle peut être entachée d'une erreur due à une pollution d'ions parasites. D'autre part, du fait que les distributions énergétiques des ions $\mathrm{Cu}^{+}$et $\mathrm{Mn}^{+}$ne sont pas homothétiques en dessous de $15 \mathrm{eV}$, le rapport $\mathrm{K}\left(\mathrm{Mn}^{+}\right) / \mathrm{K}\left(\mathrm{Cu}^{+}\right)$des émissions des ions lents d'un alliage $\mathrm{CuMn}$ de concentration donnée, dépend de la bande d'énergie fixée par la largeur de la fente de sélection. Ceci va se traduire par une translation de la courbe $a$ en fonction de la bande d'énergie des ions analysés. La courbe $b$, relative à l'émission des ions rapides d'énergie supérieure à $30 \mathrm{eV}$, ne dépend pas au contraire de l'ouverture de la fente de sélection. On peut donc considérer que la contante ionique, $k_{\text {CuMn }}=7,1$, déduite de cette courbe, est caractéristique de l'émission des alliages CuMn.

La constante ionique d'un alliage mesure le rapport des rendements en ions des deux éléments qui le composent, ramenés à $100 \%$ de concentration. Dans le cas présent, le rapport des émissions en ions rapides du manganèse au cuivre est égal à 7,1 . Si l'on se reporte au tableau I, le rapport des émissions des ions rapides, d'énergie supérieure à $30 \mathrm{eV}$, du manganèse pur au cuivre pur est de 2,8. Comme l'émission du cuivre dans l'alliage, CuMn n'est pas modifiée par la présence d'un soluté en très faible concentration, on en conclut que l'émission du manganèse est exaltée d'un facteur $7,1 / 2,8 \sim 2,5$ lorsqu'il est en solution dans le cuivre. Cet effet d'exaltation de l'émission d'une impureté dissoute dans un alliage a été fréquemment ob- 
servé [10]-[18]-[19] ; une interprétation de ce phénomène sera présentée dans un prochain article.

Des impuretés de transition ont été dissoutes dans les quatre matrices de fer, de cobalt, de nickel et de cuivre. Entre 0 et $15 \mathrm{eV}$, les distributions énergétiques des ions d'impuretés du groupe des ferromagnétiques et du cuivre sont les mêmes que celles données par les métaux purs correspondants. Par contre, nous avons toujours constaté que la distribution énergétique des ions émis par les éléments du début de la série Ti $\mathrm{V}, \mathrm{Cr}, \mathrm{Mn}$, en solution dans une des quatre matrices, était différente de celle des ions des métaux purs de ce groupe. Ces constatations résultent de la comparaison des émissions normalisées de basse énergie, $r_{\mathrm{B}}$ et $r_{\mathrm{B}}^{a}$, des ions provenant d'un élément de transition $\mathrm{B}$ pris respectivement à l'état pur et en solution dans un alliage $A_{1-C} B_{C}$ (Fig. 14). Rappelons que

$$
r_{\mathrm{B}}^{a}=\frac{k_{\mathrm{A} \mathbf{B}}^{0}}{k_{\mathrm{AB}}^{E}} r_{\mathrm{A}}
$$

où $r_{\mathrm{A}}$ est l'émission normalisée de basse énergie de la matrice, identique à celle du métal pur et $k_{\mathrm{AB}}^{E}$ la constante ionique mesurée en prenant les ions $\mathrm{B}^{+}$ dans la partie homothétique des distributions énergétiques $(E>30 \mathrm{eV})$. La similitude des distributions

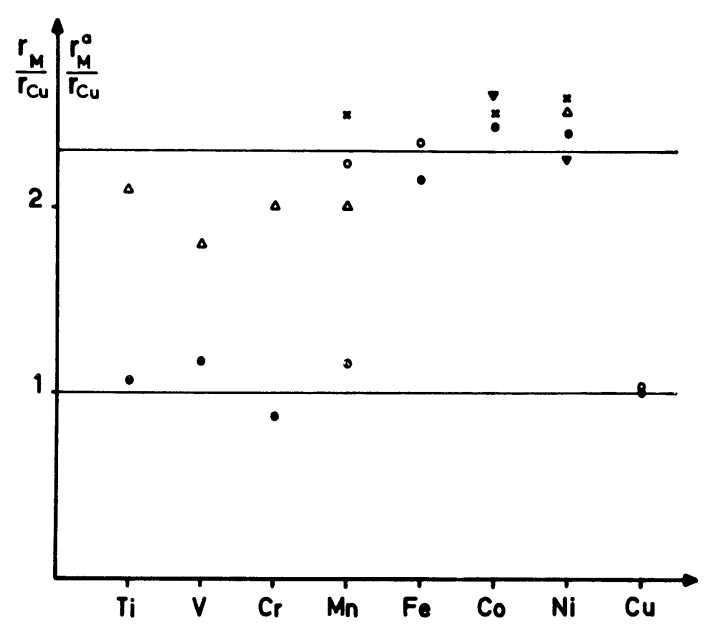

FIG. 14. - Rendements normalisés de basse énergie $r_{\mathrm{M}} / r_{\mathrm{Cu}}$ des éléments de transition à l'état pur et rendements normalisés de basse énergie

$$
\frac{r_{M}^{a}}{r_{\mathrm{Cu}}}=\frac{\mathbf{K}_{\mathrm{C}}^{0}\left(\mathrm{M}^{+}\right) / \mathrm{K}^{0}\left(\mathrm{Cu}^{+}\right)}{\mathrm{K}^{100}\left(\mathbf{M}^{+}\right) / \mathrm{K}^{100}\left(\mathrm{Cu}^{+}\right)}
$$

de ces éléments en solution

Métaux purs ; $\triangle$ Matrice de cuivre; $\bigcirc$ Matrice de nickel ; $\times$ Matrice de cobalt; $\nabla$ Matrice de fer.

énergétiques des éléments ferromagnétiques purs et alliés se traduit par la conservation de l'émission normalisée de basse énergie $r_{\mathrm{B}}^{a} / r_{\mathrm{Cu}} \sim 2$, 3. Au contraire, le changement intervenu dans le spectre d'énergie des ions émis par les éléments $\mathrm{Ti}, \mathrm{V}, \mathrm{Cr}, \mathrm{Mn}$ en solution est marqué par le fait que $r_{\mathrm{B}}^{a} \sim 2 r_{\mathrm{B}}$. On a pris l'exemple de l'émission des ions $\mathrm{Cr}^{+}$dans le métal pur et dans un alliage $\mathrm{CuCr}$ à 500 ppm de chrome, pour illustrer le changement des distributions énergétiques (Fig. 15). En prenant un rapport d'homothétie égal à l'unité pour la partie de haute énergie de ces deux distributions, on observe une croissance de l'émission du chrome plus rapide dans l'alliage que dans le métal pur, à mesure que l'énergie des ions diminue. Par

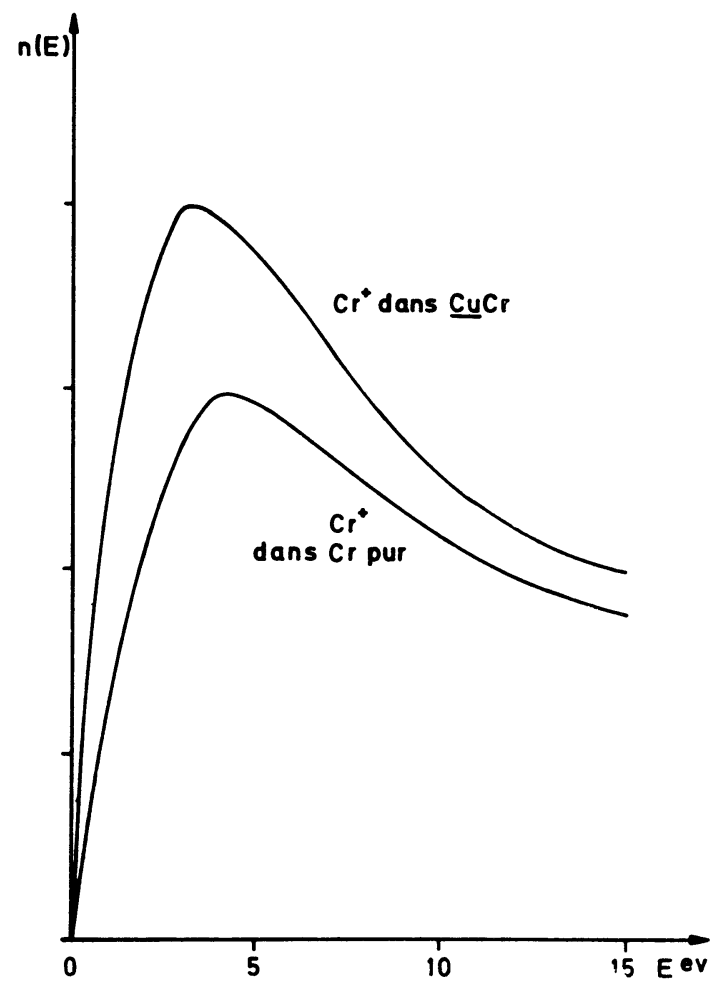

Fig. 15. - Distributions énergétiques, normalisées à $100 \mathrm{eV}$, des ions $\mathrm{Cr}^{+}$dans le chrome pur et dans l'alliage $\underline{\mathrm{CuCr} a ̀ ~} 500 \mathrm{ppm}$ de chrome.

ailleurs, l'énergie la plus probable est plus faible dans l'alliage. Ces remarques rappellent celles qui ont été faites à propos des distributions énergétiques des ions $\mathrm{Cu}^{+}$et $\mathrm{Ni}^{+}$(Fig. 4). Comme par ailleurs, les spectres énergétiques des ions $\mathrm{Cr}^{+}$et $\mathrm{Cu}^{+}$sont pratiquement identiques, on voit qu'il y a une analogie entre le spectre des ions $\mathrm{Cr}^{+}$de l'alliage $\underline{\mathrm{Cu}} \mathrm{Cr}$ et celui des ions $\mathrm{Ni}^{+}$.

Il semble donc que l'émission des ions de basse énergie émis par les éléments d'un même groupe $\mathbf{T i}$, $\mathrm{V}, \mathrm{Cr}, \mathrm{Mn}$ en solution dans l'un des autres métaux de la série $\mathrm{Cu}, \mathrm{Ni}, \mathrm{Co}, \mathrm{Fe}$ soit caractérisée par une distribution énergétique qui ressemble fortement à celle des ions ferromagnétiques. Il a là un comportement d'ensemble de ces éléments qu'il faut certainement attribuer au processus d'ionisation lui-même. $\mathrm{Si}$ l'on reprend l'hypothèse d'une destruction des états auto-ionisants, avancée pour expliquer les distributions des ions $\mathrm{Cu}^{+}$et $\mathrm{Ni}^{+}$des métaux purs, il faut s'attendre à une certaine analogie entre les processus d'ionisation des éléments $\mathrm{Ti}, \mathrm{V}, \mathrm{Cr}, \mathrm{Mn}$ en solution et le groupe des éléments ferromagnétiques. 
5. Conclusion. - Cette étude apporte des précisions sur les aspects généraux des distributions en énergie des ions d'une même espèce, en même temps qu'elle met en relief le caractère particulier de l'émission des ions de basse énergie d'un élément.

Ainsi, par exemple, on peut dire d'une manière générale que la distribution des ions simples $\mathbf{M}^{+}$ présente toujours un maximum très net, situé vers $4 \mathrm{eV}$ et suivi d'une décroissance assez lente. Par contre, le spectre d'énergie des ions moléculaires ne s'étend guère au-delà d'une vingtaine d'électrons-volts avec un maximum situé vers $5 \mathrm{eV}$. Ces constatations corroborent des mesures d'énergie moyenne, obtenues par ailleurs, pour ces deux espèces d'ions [2] : énergie moyenne élevée pour les ions simples et faible pour les ions moléculaires.

Nous avons mis l'accent, au cours de cette étude, sur certains aspects particuliers de l'émission des ions de basse énergie, notamment en ce qui concerne les ions $\mathrm{M}^{+}$des éléments de transition. Le fait, par exemple, que les distributions énergétiques des ions $\mathrm{Cu}^{+}$et $\mathrm{Ni}^{+}$émis par les métaux purs soient différentes, est certainement imputable aux mécanismes d'ionisations. Il en est de même pour les ions $\mathrm{Ti}^{+}$, $\mathrm{V}^{+}, \mathrm{Cr}^{+}, \mathrm{Mn}^{+}$qui ne présentent pas la même distribution selon qu'ils proviennent de métaux purs ou d'alliages. Nous avons établi dans ce domaine un ensemble de résultats expérimentaux qui sera analysé dans un prochain article.

\section{Bibliographie}

[1] Jurela, Z. et Perovic, B., Can. J. Phys. 46(1968) 773.

[2] Hennequin, J.-F., J. Physique 29 (1968) 655-957.

[3] Blaise, G. et Slodzian, G., J. Physique 31 (1970) 93.

[4] Castaing, R., Hennequin, J.-F., Henry, L. et Slodzian, G., Focusing of charged particles II 4 (1967) 161.

[5] Slodzian, G., Annls de Phys. 9 (1964) 591.

[6] Blaise, G., Thèse, Orsay (1972).

[7] Leleyter, M., Slodzian, G., Quettier, R., Revue Phys. Appl. 7 (1972) 117.

[8] Rivière, J. C., Proc. Phys. Soc. London 70 (1957) 676.

[9] Blaise, G., Slodzian, G., C. R. Hebd. Séan. Acad. Sci. B 271 (1970) 1216.

[10] Beske, H. E., Z. Naturforsch. a 22 (1967) 459.
[11] Jurela, Z., Atomic Collision Phenomena in Solids, Amsterdam (1970).

[12] Joyes, P., J. Physique 29 (1968) 774.

[13] Echsner, H., Z. Phys. 238 (1970) 433.

[14] Joyes, P. et Leleyter, M., J. Phys. $B$ (à paraître).

[15] Bernheim, M., Blaise, G. et Slodzian, G., Int. J. Mass Spectr. Ion Phys. 8 (1972) 001.

[16] Woodyard, J. R., ASTMS, 1965.

[17] Hernandez, R., Lanusse, P., Slodzian, G., $C$. $R$. Hebd. Séan. Acad. Sci. B 271 (1970) 1033.

[18] Blaise, G., Slodzian, G., C. R. Hebd. Séan. Acad. Sci. B 273 (1971) 357.

[19] Shelten, J., Z. Naturforsch. a 23 (1968) 109. 